TITLE: Health Care Disparities in Radiology: A Primer for Resident Education

Authors:

Lisa Americo, $\mathrm{BS}^{\mathrm{a}}$

${ }^{\mathrm{a} C U N Y}$ School of MedicinelThe Sophie Davis Biomedical Education Program

Manhattan, NY

Amit Ramjit, MD ${ }^{\mathrm{b}}$

${ }^{\mathrm{b}}$ Zucker School of Medicine at Hofstra Northwell, Staten Island University Hospital

Staten Island, NY

Michelle $\mathrm{Wu}, \mathrm{MD}^{\mathrm{c}}$

${ }^{c}$ Zucker School of Medicine at Hofstra Northwell, Staten Island University Hospital

Staten Island, NY

Drew Caplin, $\mathrm{MD}^{\mathrm{e}}$

${ }^{\mathrm{e}}$ Zucker School of Medicine at Hofstra Northwell, Northwell Health Physician Partners

Manhasset, NY

Joseph Mazzie, DO ${ }^{\mathrm{f}}$

${ }^{\mathrm{f}}$ NYU-Winthrop Department of Radiology

Mineola, NY

Leif Jensen, MD MPH ${ }^{\mathrm{d}}$

${ }^{\mathrm{d}}$ University of Utah School of Medicine

Salt Lake City, UT

Maitray D. Patel, MD

${ }^{\mathrm{j}}$ Mayo Clinic Arizona

Phoenix, AZ

James Milburn, MD ${ }^{\mathrm{h}}$

${ }^{\mathrm{h}}$ Ochsner Clinic

New Orleans, LA

Christopher P. Ho, MD ${ }^{\mathrm{i}}$

${ }^{\mathrm{i}}$ Emory University School of Medicine

Atlanta Georgia

This is the author's manuscript of the article published in final edited form as:

Americo, L., Ramjit, A., Wu, M., Jensen, L., Caplin, D., Mazzie, J., ... Sarkany, D. (2018). Health Care Disparities in Radiology: A Primer for Resident Education. Current Problems in Diagnostic Radiology. 
Glenn C. Gaviola, MD $^{\mathrm{g}}$

${ }^{\text {g} B r i g h a m ~ a n d ~ W o m e n ' s ~ H o s p i t a l ~}$

Boston, MA

Carolynn M. DeBenedectis, $\mathrm{MD}^{\mathrm{k}}$

${ }^{\mathrm{k}}$ University of Massachusetts Medical School

Worcester, MA

Darel E. Heitkamp, MD ${ }^{1}$

${ }^{1}$ Indiana University School of Medicine

Indianapolis, IN

Corresponding author - David Sarkany, MD MS-HPEd ${ }^{\mathrm{m}}$

${ }^{\mathrm{m}}$ Zucker School of Medicine at Hofstra Northwell

Staten Island University Hospital Northwell Health

Department of Radiology

475 Seaview Avenue

Staten Island, NY 10305 USA

Phone: 718-226-8297

Fax: 718-226-8335

dsarkany@northwell.edu or ids_1@hotmail.com

No author has a conflict of interest

\section{Abstract Primer}

As the population of the United States grows increasingly diverse, health care disparities become vital to understand and mitigate. The ethical and financial implications of how groups of Americans gain access to health care have evolved into some of today's most challenging socioeconomic problems. Educators in radiology are just beginning to tackle the concepts of health care disparities, unconscious bias and cultural competency.

In July 2017, the Accreditation Council for Graduate Medical Education required that all trainees and teaching faculty of accredited training programs receive training and experience in new areas of quality improvement to include an understanding of health care disparities as part 
of the core competencies. To our knowledge, there is no centralized curriculum regarding health care disparities for radiology residents and fellows. Many programs, in fact, have yet to introduce the concept to their trainees, who may have difficulty recognizing that this is even a problem affecting radiology. This manuscript serves as a primer for radiology trainees on health care disparities, with the goal of defining major concepts and providing examples of how variable access to radiological care can have substantial impact on patient outcomes. 


\section{INTRODUCTION}

In July 2017, the Accreditation Council for Graduate Medical Education (ACGME) updated the Common Program Requirements for residency training programs, adding initiatives in patient safety, quality, and physician well-being. Under the new requirements for quality, the ACGME included education in a much-needed knowledge domain: health care disparities (1). Ask a radiology resident about this topic and you're bound to receive a puzzled look. Since most residents work and learn in reading rooms isolated from direct patient contact, many feel that culture has little influence on how they interpret images. Indeed, many trainees may not think about health care disparities occurring in the field of radiology or even know what the term “disparities" means. Educators could easily get a sense for baseline trainee knowledge by simply asking their residents: "Do health care disparities exist in the field of radiology, and if so, how do they impact patients?" The aim of this article is to demonstrate how educators might teach trainees about health care disparities by focusing on those that affect patients receiving care in radiology.

\section{IMPORTANCE}

As educators, we need to be deliberate in assigning importance to this topic. Residents tend to believe that health care disparities only manifest in fields such as internal medicine, pediatrics, and surgery, when in reality they occur in all areas of health care delivery. The fact of the matter is that radiology services are expensive and many patients simply cannot afford our expertise. Trainees should be able to identify economic and social differences which could result in health care disparities from social media, the news, and even everyday situations. Inequalities are common in both urban and rural settings across the United States, affecting ethnic and racial 
minorities as well as those of lower socioeconomic status. Other criteria known to place groups at risk for health care disparities focus on age, gender, religion, and disability (2).

Four concepts underscore the importance of teaching health care disparities in today's residency curriculum:

1. The Institute of Medicine (IOM) reported in 2002 that bias may contribute to health care disparities.

2. By the year $2060,57 \%$ of the American population will be made up of underrepresented groups subject to health care disparities (3).

3. In 2009, the Joint Center for Political and Economic Studies reported that if health care disparities did not exist between 2003-2006 alone, \$229 billion in direct medical expenses and over $\$ 1$ trillion in indirect expenses would have been saved (4).

4. A vital component of the solution of health care disparities is education in cultural competency and unconscious bias, addressing the underlying causes of disparities and discussing strategies to improve diversity in our workforce (5).

\section{DEFINITIONS}

In order to establish a baseline understanding for residents, it is necessary to carefully define the terms health care disparity, cultural competency, and unconscious bias. Health care disparities can be defined as differences in the incidence and prevalence of detrimental conditions and outcomes experienced by certain populations when compared with the national average $(6,7)$. Within the United States, commonly cited disparities include differences in incidence of cervical cancer, breast cancer fatalities, and cancer management $(7,8)$. What causes health disparities to appear in certain populations? Numerous factors propagate health 
disparities, such as lack of access to health care, education, employment, lifestyle choices, and the way different groups of patients are treated by medical professionals (8). For example, patients that rely on public transportation may find themselves missing appointments because they cannot always find a reliable means of travel. Patients with low health literacy may not understand their physicians' recommendations (6).

Cultural competency can be understood as behavior, attitudes, and beliefs that guide physicians to work effectively in cross-cultural situations. A physician may encounter patients who understand limited English and whose cultures may express their discomfort in a manner different from what the physician may be accustomed. Ignoring these nuances would be exhibiting poor cultural competency, which can result in subpar patient care $(6,7)$. By exercising cultural competency, however, it is possible for physicians to provide optimal care to all patients. Residents must not underestimate the influence of culture on both their patients' behavior and their own clinical decision making. Cultural competency should serve to further reinforce the important interplay of beneficence and autonomy in medical ethics.

The last concept to address is that of unconscious bias. Unconscious bias, also called implicit bias, refers to the stereotypes that affect our behaviors and interactions with others in a subconscious manner. Unconscious bias relies on assumptions about individuals and their related groups to guide our actions (5). They occur without our control and cannot be altered through conscious thought. For example, it has been described in various fields of science that faculty are biased to gender (showing preference for males) (9). For efficiency, our minds are naturally attuned to quickly assess people involuntarily by making connections between individuals and 
certain attributes we may believe about them. Unsurprisingly, society and various hierarchies guide what attributes we value and what attributes we disdain. While unconscious bias is a known contributor to health care disparities, cultural competency is an effective means by which to help mitigate it. Educating trainees in a way such as to reduce unconscious bias and improve cultural competency stands to be an effective means for radiology educators to combat health care disparities (5).

\section{RADIOLOGY LITERATURE RECOGNIZES HEALTH CARE DISPARITIES}

Armed with just the basic terminology, residents can explore the literature to understand firsthand how health care disparities are experienced in radiology. These examples are not meant to serve as a comprehensive literature review, but rather an experience to introduce residents to the presence of health care disparities in radiology.

In a 2013 study of 149 patients with mammogram-detected breast cancer residing in Chicago, Rauscher et al. found that $46 \%$ had a potentially detectable suspicious breast finding on a prior mammogram that was originally read as non-malignant. The proportion of missed malignant lesions was greater among minority patients ( $54 \%$ vs $39 \%$ ). Additionally, missed detection was greater in patients with incomes below $\$ 30,000(65 \%$ vs $36 \%)$, patients with less education ( $58 \%$ vs $39 \%)$, and patients without private health insurance ( $63 \%$ vs $40 \%)$. These findings were all significant, and the paper suggested higher rates of false negative results on screening mammography in patients who were socioeconomically disadvantaged (10).

In another published example of health care disparities, a retrospective review from April 2015 to March 2016 evaluated the impact of race and socioeconomic status on what researchers 
described as "imaging missed care opportunities" or IMCOs at a quaternary care center with associated outpatient centers. The review defined IMCOs as imaging appointments or studies not performed on their scheduled dates and times. A total of 57,847 patients with a total of 89,943 scheduled unique imaging appointments were reviewed. Of these appointments, $6.1 \%$ were marked as IMCOs. Both race and socieconomic status were independent predictors of IMCOs. Patients with median household incomes of less than $\$ 50,000$ were more likely to have IMCOs compared to patients with incomes of $\$ 100,000$ or more. Results also showed higher rates of IMCOs in non-English speakers and minorities. The authors proposed multiple causal factors including limited financial means and geographic barriers to access (11).

To address and combat patient care disparities, a move toward physician diversity has become a priority for many health delivery organizations. Our increasingly diverse population requires an equally diverse radiology workforce, as diversity among health care providers is believed to be a crucial element to improving equal access to health care in the United States (12). Previous research has found that students from medical schools with higher levels of diversity feel more confident when working with culturally diverse patients. Additionally, medical students from underrepresented backgrounds were shown more likely to choose careers that include service to the underserved (5). Workforce diversity has also been shown to enhance creativity, productivity, teamwork, and innovation $(13,14)$.

Diversity in medicine is of paramount importance in radiology, as there is a distinct shortage of women and minorities in the field (13). In 2011, despite comprising $48.3 \%$ of all students enrolled at US medical schools, women represented only $27.8 \%$ of applicants to diagnostic radiology residencies (15). Similarly, in 2009, underrepresented minorities accounted for $15.3 \%$ of medical student graduates, but only $6.5 \%$ of practicing radiologists (13). If 
improving the diversity of providers is an essential component to improving health care disparities, radiology indeed has its work cut out for it. Resident education on this topic may be the most effective first step we can take.

These examples cover a wide variety of disparity issues in radiology and clearly illustrate a real impact on the field. Not only do health care disparities occur in areas of patient centered radiology such as mammography, but as the topic of IMCOs demonstrates, it affects all areas of radiology where imaging appointments are scheduled. In addition, the call for workplace diversity to combat health care disparities is as important and impactful for radiology as any other field.

\section{TEACHING OUR TRAINEES}

Most residency directors have yet to formally introduce the topic of healthcare disparities into their trainee curricula, as the subject is still being developed both locally and nationally. Residents may notice hospitals and health care organizations mandating that their employees receive education in cultural competency, and some may be under the misguided belief that these concepts are not relevant to radiologists. The goal of this primer is to serve as a starting point for local resident discussion regarding healthcare disparities in radiology and the concepts of cultural competency and unconscious bias.

Curricula in these domains have already been developed by other organizations, such as primary care residencies, governmental agencies, and medical schools $(16,17,18)$. These resources and many others can be used as a foundation to create a formal universal radiology trainee curriculum. It is the goal of the above authors, members of the Association of Program Directors in Radiology Academic Output Task Force and Common Program Requirements Ad 
Hoc Committee, to begin developing such a curriculum for use by all radiology trainees and educators.

\section{CONCLUSION}

Disparities occur widely throughout health care, and radiology is certainly no exception. However, it is a relatively new concept in radiology graduate medical education, and most residents do not expect to engage in discussion on this topic. Many residents may have a hard time believing that there is a problem with health disparities in radiology and may wish to utilize their conference time to improve their interpretive skills rather than discussing access to health care. The topic, however, is of vital importance for radiologists to understand, for while our specialty works toward patient-centered care, our workforce is not truly aware of health care disaprities and is not optimally diversified. This paper's review of health care disparities, definition of key terms, and provision of real radiology examples will hopefully serve as a starting point to engage trainees in a broader discussion of this important topic, and serve as our foundation for future currcular deyelopment

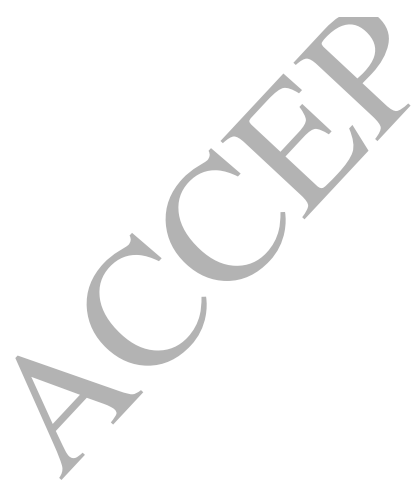


References:

1. Available at www.acgme.org -

http://www.acgme.org/Portals/0/PFAssets/ProgramRequirements/CPRs_Section\%20

$\underline{\text { VI_with-Background-and-Intent_2017-01.pdf; accessed on January 14, } 2018}$

2. Jordan J, Lightefoote J. Overcoming health disparities in the United States: The value imperative for healthier populations. Journal of the American College of Radiology, article in press.

3. Maldonado $\mathrm{M}$, et al. The role that graduate medical education must play in ensuring health equity and eliminating health care disparities. Annals of the American Thoracic Society 2014; 11: 603-607.

4. LaVeist T, et al. The economic burden of health inequalities in the United States. Joint Center for Political and Econormic Studies, September 2009.

5. Allen BJ, Garg K. Diversity matters in academic radiology: Acknowledging and addressing unconscious bias. J Am Coll Radiol. 2016;13(12):1426-1432.

6. Brusin JH. How cultural competency can help reduce health disparities. Radiol Technol. 2012;84(2):129-147.

7. Matthews-Juarez P, Juarez PD. Cultural competency, human genomics, and the elimination of health disparities. Soc Work Public Health. 2011;26(4):349-365.

8. Gunderman RB. Addressing Racial and Ethnic Disparities in Health Care. Radiology. 2007;244(1):28-30.

9. Moss-Racusin CA, Dovidio JF, Brescoll V, et al. Science faculty's subtle gender biases favor male students. Proc Natl Acad Sci U S A 2012; 109:16474-9. 
10. Rauscher GH, Khan JA, Berbaum ML, Conant EF. Potential missed detection with screening mammography: Does the quality of radiologist's interpretation vary by patient socioeconomic advantage/disadvantage. Ann Epidemiol. 2013;23(4):210-214.

11. Glover M, Daye D, Khalilzadeh O, et al. Socioeconomic and demographic predictors of missed opportunities to provide advanced imaging services. J Am Coll Radiol. Article in press.

12. Institute of Medicine. In the nation's compelling interest: ensuring diversity in the health care workforce. Washington, District of Columbia: National Academy of Sciences; 2004.

13. Lightfoote JB, Fielding JR, Deville C, et al. Improving diversity, inclusion, and representation in radiology and radiation oncology part 1: why these matter. J Am Coll Radiol 2014; 11:673-80.

14. Hewlett SA, Marshall M, Sherbin L. How diversity can drive innovation. Harv Bus Rev 2013. December:30

15. Higgins MC, Hwang W-T, Richard C, et al. Underrepresentation of women and minorities in the united states IR academic physician workforce. J Vasc Interv Radiol. 2016; $27: 1837-1844$.

16. Ayailable at www.gnyha.org - https://www.gnyha.org/tool/context-and-theory-ofsocial-determinants-of-health-powerpoint/; accessed on April 24, 2018

17. Available at www.gnyha.org - https://www.gnyha.org/tool/training-primary-care$\underline{\text { residents-on-the-social-determinants-of-health/; accessed on April 24, } 2018}$ 
18. Vela MB, Kim KE, Tang H, and Chin MH. Innovative health care disparities curriculum for incoming medical students. J Gen Intern Medicine. 2008; 23: 10281032.

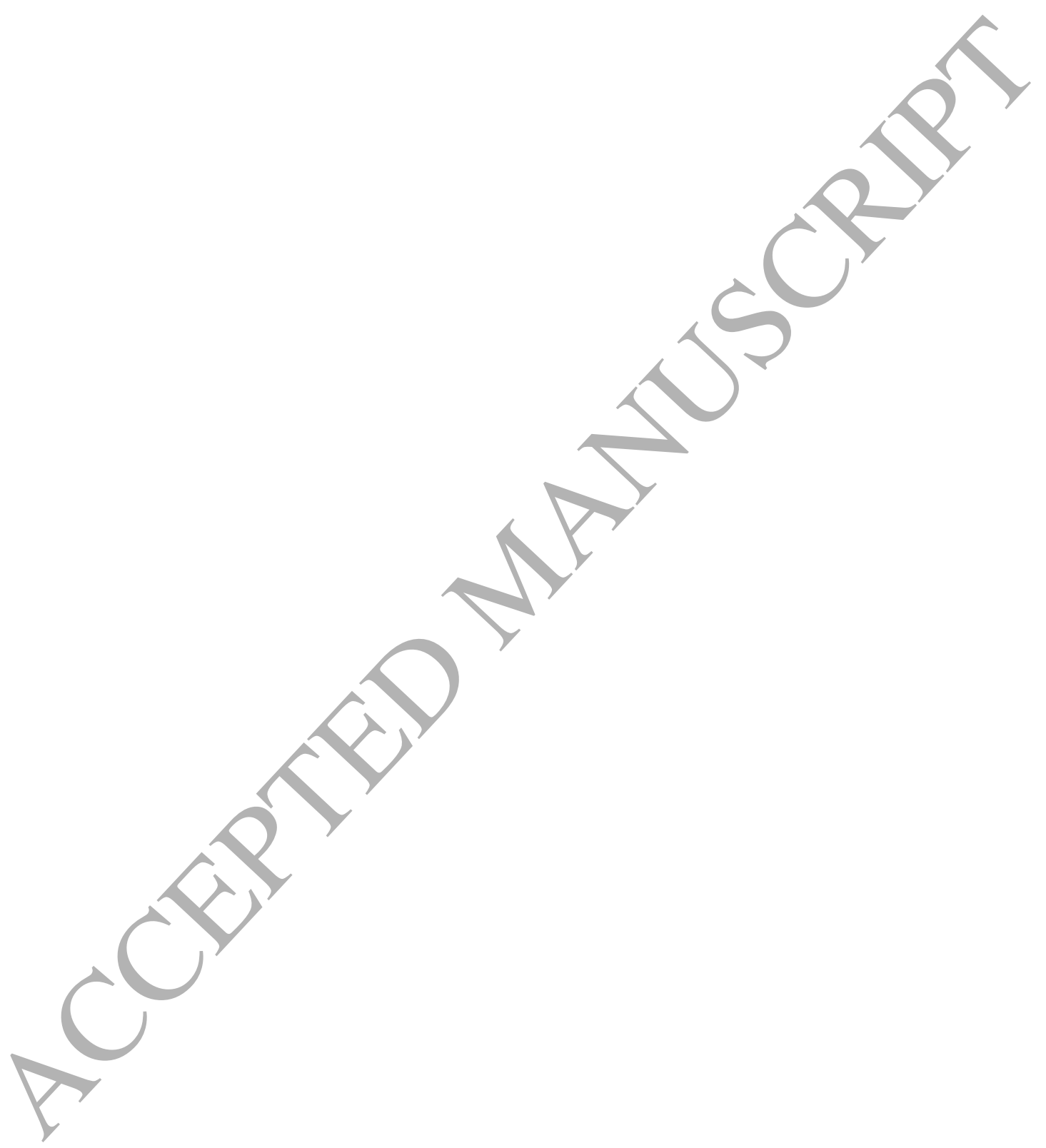

